\title{
The faint-end of galaxy luminosity functions at the Epoch of Reionization
}

\author{
B. Yue ${ }^{1}$, M. Castellano ${ }^{2}$, A. Ferrara ${ }^{3,4}$, A. Fontana ${ }^{2}$, E. Merlin ${ }^{2}$, \\ R. Amorín ${ }^{5,6}$, A. Grazian ${ }^{2}$, E. Mármol-Queralto ${ }^{7}$,
} M. J. Michałowski ${ }^{8}$ A. Mortlock ${ }^{7}$, D. Paris ${ }^{2}$, S. Parsa ${ }^{7}$, S. Pilo ${ }^{2}$, P. Santini ${ }^{2}$ and M. Di Criscienzo ${ }^{2}$

${ }^{1}$ National Astronomical Observatories, Chinese Academy of Sciences, 20A, Datun Road, Chaoyang District, Beijing, 100012, China email: yuebin@bao.ac.cn

${ }^{2}$ INAF - Osservatorio Astronomico di Roma, Via Frascati 33, I-00078 Monte Porzio Catone $(\mathrm{RM})$, Italy

${ }^{3}$ Scuola Normale Superiore, Piazza dei Cavalieri 7, I-56126 Pisa, Italy

${ }^{4}$ Kavli IPMU (WPI), Todai Institutes for Advanced Study, the University of Tokyo, Japan

${ }^{5}$ Cavendish Laboratory, University of Cambridge, 19 JJ Thomson Avenue, Cambridge, CB3 OHE, UK

${ }^{6}$ Kavli Institute for Cosmology, University of Cambridge, Madingley Road, Cambridge, CB3 OHA, UK

${ }^{7}$ SUPA, Scottish Universities Physics Alliance, Institute for Astronomy, University of Edinburgh, Royal Observatory, Edinburgh, EH9 3HJ, U.K.

${ }^{8}$ Astronomical Observatory Institute, Faculty of Physics, Adam Mickiewicz University, ul. Słoneczna 36, 60-286 Poznań, Poland

\begin{abstract}
During the Epoch of Reionization (EoR), feedback effects reduce the efficiency of star formation process in small halos or even fully quench it. The galaxy luminosity function (LF) may then turn over at the faint-end. We analyze the number counts of $z>5$ galaxies observed in the fields of four Frontier Fields (FFs) clusters and obtain constraints on the LF faint-end: for the turn-over magnitude at $z \sim 6, M_{\mathrm{UV}}^{\mathrm{T}} \gtrsim-13.3$; for the circular velocity threshold of quenching star formation process, $v_{c}^{*} \lesssim 47 \mathrm{~km} \mathrm{~s}^{-1}$. We have not yet found significant evidence of the presence of feedback effects suppressing the star formation in small galaxies.
\end{abstract}

Keywords. galaxies: high-redshift, gravitational lensing

\section{Overview}

During the Epoch of Reionization (EoR), the feedback effects suppress the formation of small galaxies, resulting in a turn-over in the luminosity function (LF) faint-end (e.g. Gnedin 2016; Ceverino et al. 2017). It is essential to check if such a turn-over exists by using the dataset of the deepest galaxy surveys (Yue et al. 2014). In blank fields, high- $z$ galaxies with absolute UV magnitude down to $M_{\mathrm{UV}} \sim-17$ (e.g. Bouwens et al. 2015) have been detected, no evidence for such a LF turn-over has ever been found. The strong gravitational lensing provides opportunity to detect even fainter EoR galaxy populations. However, even though the LF turn-over is still not yet apparent (Atek et al. 2015a,b; Castellano et al. 2016a; Livermore et al. 2017; Ishigaki et al. 2017; Laporte et al. 2016; Bouwens et al. 2017).

We have analyzed the number counts of high- $z(z>5)$ galaxy samples in ASTRODEEP catalogs (Castellano et al. 2016b; Merlin et al. 2016; Di Criscienzo et al. 2017) of the four FFs (Lotz et al. 2017) clusters: Abell 2744 (A2744), MACSJ0416.1-2403 (M0416), MACSJ0717.5+3745 (M0717) and MACSJ1149.5+2223 (M1149). It is found that the 


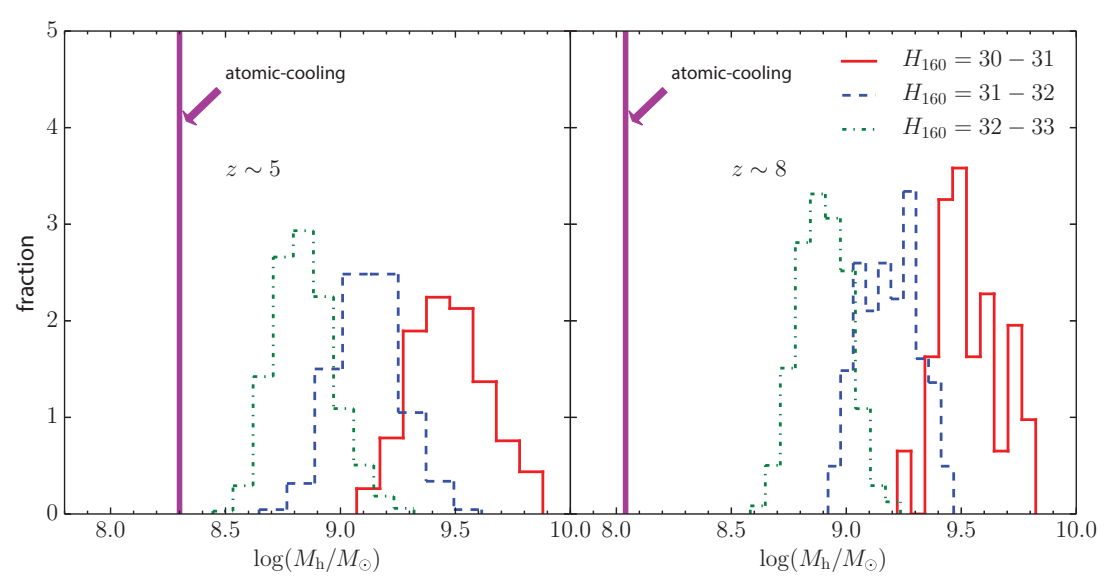

Figure 1. The mass distribution of host halos of galaxies with different apparent magnitudes at $z \sim 5$ and $z \sim 8$.

number counts reconstructed by using different lensing models $\dagger$ are basically consistent with each other at the $H_{160} \lesssim 31$, where $H_{160}$ is the reconstructed H-band intrinsic apparent magnitude. However at $H_{160} \gtrsim 31$ there are obvious discrepancies. To reduce the uncertainties, in each magnitude bin we adopt the median of the galaxy numbers reconstructed using different lensing models. In the median number count, galaxies as faint as $H_{160} \sim 34$ are detected. According to galaxy formation simulations (Salvaterra et al. 2011), their host halos are around $\sim 10 \times$ the atomic-cooling threshold, see Fig. 1 .

We compared the number counts with the predictions from Monte Carlo simulations that use given LF model as inputs, to obtain the constraints on the LF turn-over and the feedback strength in a LF model with feedback effects (Yue et al. 2016) during the EoR (Castellano et al. 2016a; Yue et al. 2017).

\section{The LF models}

We investigate two LF models:

- In the empirical model the LF approaches the Schechter formula when the $M_{\mathrm{UV}}$ is much smaller than a "turn-over" magnitude $M_{\mathrm{UV}}^{\mathrm{T}}$, and drops rapidly when $M_{\mathrm{UV}}$ is larger than $M_{\mathrm{UV}}^{\mathrm{T}}$,

$$
\Phi\left(M_{\mathrm{UV}} \mid M_{\mathrm{UV}}^{*}, \Phi^{*}, \alpha, M_{\mathrm{UV}}^{\mathrm{T}}\right)=\Phi_{\mathrm{Sch}}\left(M_{\mathrm{UV}} \mid M_{\mathrm{UV}}^{*}, \Phi^{*}, \alpha\right) \times 0.5\left[\left(1-\operatorname{erf}\left(M_{\mathrm{UV}}-M_{\mathrm{UV}}^{\mathrm{T}}\right)\right]\right.
$$

where $\Phi_{\text {Sch }}$ is the standard Schechter LF. The model has three Schechter parameters $M_{\mathrm{UV}}^{*}, \Phi^{*}$ and $\alpha$, and one more parameter $M_{\mathrm{UV}}^{\mathrm{T}}$.

- In the physically-motivated model, we first calibrate a $z$-dependent star formation efficiency - halo mass relation (Mason et al. 2015) by using observed LF at $z_{0}=5$, then derive the LFs at any other redshifts according to halo assemble histories. Considering feedback effects during the EoR, the star formation process is quenched in halos with circular velocity $v_{c}$ below a threshold $v_{c}^{*}$ and located in ionized bubbles. In Fig. 2 we show the LFs for various $f_{\text {esc }}$ and $v_{c}^{*}$. The number of star-forming galaxies decreases fast when $v_{c}<v_{c}^{*}$, however there are still galaxies with relic stars shine light at the faintest-end.

\section{$\dagger$ https://archive.stsci.edu/prepds/frontier/lensmodels/}




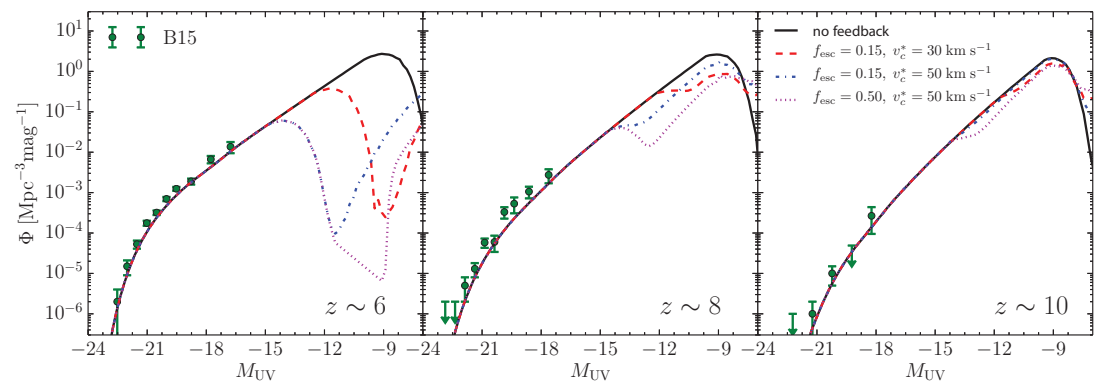

Figure 2. The LFs in the physically-motivated model for various $f_{\text {esc }}$ and $v_{c}^{*}$, at $z \sim 6,8$ and 10 respectively.
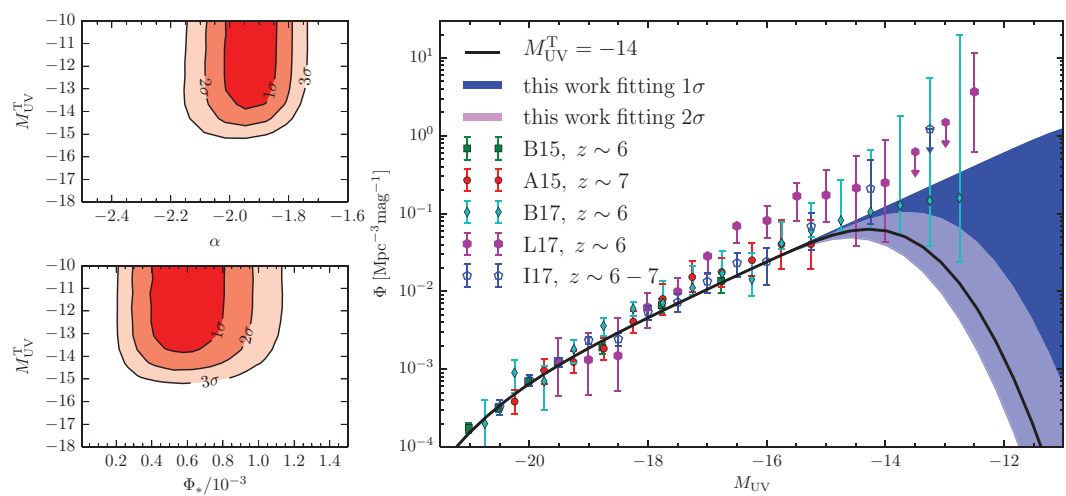

Figure 3. The constraints on the parameters $\alpha, \Phi^{*}$ and $M_{\mathrm{UV}}^{\mathrm{T}}$ for the empirical LF model at $z \sim 6$ (left), and the corresponding LF (right). In the right panel we plot the observations of Bouwens et al. (2015); Atek et al. (2015a); Bouwens et al. (2017); Livermore et al. (2017) and Ishigaki et al. (2017) respectively.

\section{The Constraints on the LF Faint-end and Discussions}

\subsection{The empirical LF model:}

In Fig. 3 we show the constraints on $\alpha, \Phi^{*}$ and $M_{\mathrm{UV}}^{\mathrm{T}}$ at $z \sim 6$ and the corresponding LF. After marginalizing $\alpha$ and $\Phi^{*}$, we obtain $M_{\mathrm{UV}}^{\mathrm{T}} \gtrsim-13.3$ ( $1 \sigma$ C.L. $)$. Obviously, the upper boundary of the $M_{\mathrm{UV}}^{\mathrm{T}}$ is still open, we have not yet found the evidence of the LF turn-over in the FFs data.

The constraint we obtain is a bit deeper than Bouwens et al. (2017) (B17) which approximately corresponds to $M_{\mathrm{UV}}^{\mathrm{T}} \gtrsim-14$ of our model, see the right panel of Fig. 3 . The different methodologies might be the reason. To exclude the number counts in extremely faint magnitude bins where only in a few lensing models there are galaxies detected, we use the median of the galaxy number counts in different lensing models, while, B17 used a forward-modeling method to incorporate the systematics. As a conservative check, we find that if we drop all galaxies with magnification $>100$ in the data, we obtain $M_{\mathrm{UV}}^{\mathrm{T}} \gtrsim-14.8$ at $1 \sigma$ C.L.

\subsection{The physically-motivated LF model:}

In the left panel of Fig. 4 we show the constraints on $f_{\text {esc }}$ and $v_{c}^{*}$ from the combination of FFs galaxy number counts and Planck2016 CMB Thompson scattering optical depth (Planck Collaboration et al. 2016). After marginalizing $f_{\mathrm{esc}}$ we obtain $v_{c}^{*} \lesssim 47 \mathrm{~km} \mathrm{~s} \mathrm{~s}^{-1}$ ( $1 \sigma$ C.L.), corresponding to halo mass $M_{\mathrm{h}} \approx 4.6 \times 10^{9} M_{\odot}$ and $1.6 \times 10^{9} M_{\odot}$ (about 20 

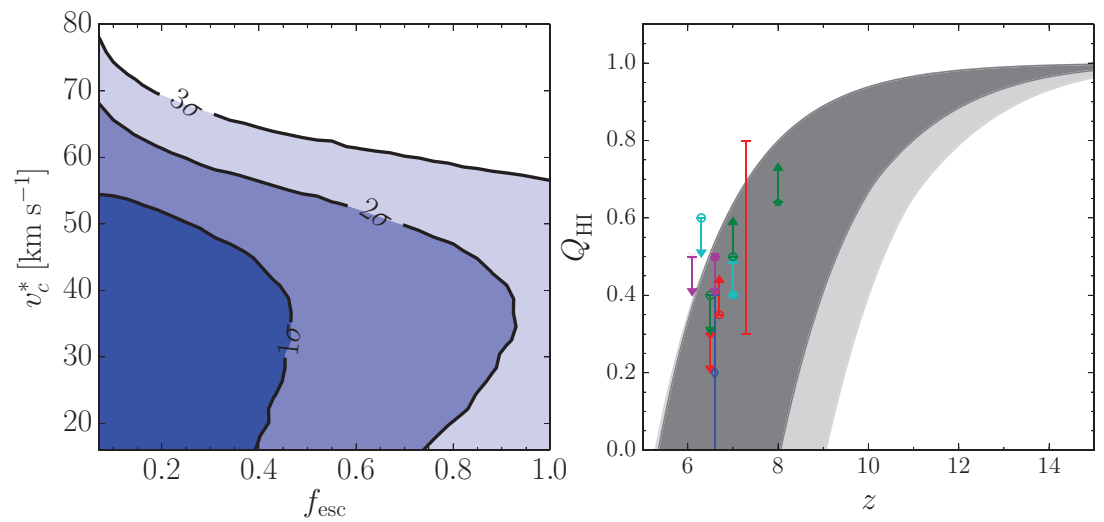

Figure 4. The constraints on parameters $f_{\text {esc }}$ and $v_{c}^{*}$ in physically-motivated LF model (left), and the corresponding reionization history (right). In the right panel, the points are observational constraints by Lyman Alpha Emitters, GRBs and QSOs observations. Original references are collected in Ota et al. (2017).

times higher than atomic-cooling threshold), or $M_{\mathrm{UV}} \approx-13.9$ and $\approx-14.8$, at $z=5$ and 10 respectively. Still, the lower boundary on $v_{c}^{*}$ is open, implying that the turn-over is not apparent.

We also show the corresponding reionization history under the constraints, see the right panel of Fig. 4. In the upcoming future, tighter constraints on the neutral fraction evolution history are expected, we will learn more about galaxies at the LF faint-end.

\section{References}

Atek, H., Richard, J., Kneib, J.-P., et al. 2015a, ApJ, 800, 18

Atek, H., Richard, J., Jauzac, M., et al. 2015b, ApJ, 814, 69

Bouwens, R. J., Oesch, P. A., Illingworth, G. D., Ellis, R. S., \& Stefanon, M. 2017, ApJ, 843, 129

Bouwens, R. J., Illingworth, G. D., Oesch, P. A., et al. 2015, ApJ, 803, 34

Castellano, M., Yue, B., Ferrara, A., et al. 2016a, ApJ, 823, L40

Castellano, M., Amorín, R., Merlin, E., et al. 2016b, A\& A, 590, A31

Ceverino, D., Glover, S. C. O., \& Klessen, R. S. 2017, MNRAS, 470, 2791

Di Criscienzo, M., Merlin, E., Castellano, M., et al. 2017, ArXiv e-prints, arXiv:1706.03790

Gnedin, N. Y. 2016, ApJ, 825, L17

Ishigaki, M., Kawamata, R., Ouchi, M., Oguri, M., \& Shimasaku, K. 2017, ArXiv e-prints, arXiv: 1702.04867

Laporte, N., Infante, L., Troncoso Iribarren, P., et al. 2016, ApJ, 820, 98

Livermore, R. C., Finkelstein, S. L., \& Lotz, J. M. 2017, ApJ, 835, 113

Lotz, J. M., Koekemoer, A., Coe, D., et al. 2017, ApJ, 837, 97

Mason, C. A., Trenti, M., \& Treu, T. 2015, ApJ, 813, 21

Merlin, E., Amorín, R., Castellano, M., et al. 2016, A\&SA, 590, A30

Ota, K., Iye, M., Kashikawa, N., et al. 2017, ApJ, 844, 85

Planck Collaboration, Adam, R., Aghanim, N., et al. 2016, A\&A, 596, A108

Salvaterra, R., Ferrara, A., \& Dayal, P. 2011, MNRAS, 414, 847

Yue, B., Ferrara, A., Vanzella, E., \& Salvaterra, R. 2014, MNRAS, 443, L20

Yue, B., Ferrara, A., \& Xu, Y. 2016, MNRAS, 463, 1968

Yue, B., Castellano, M., Ferrara, A., et al. 2017, ArXiv e-prints, arXiv:1711.05130 